HRJ

V.2 n.11 (2021)

Recebido: $15 / 08 / 2020$

Aceito: 20/04/2021

\title{
Cuidados paliativos para idosos frágeis durante a pandemia do Coronavírus
}

\author{
Rafael Spíndola do Prado ${ }^{1}$ \\ Karine Marques Costa dos Reis ${ }^{2}$ \\ Silvia Maria Gonçalves Coutinho ${ }^{3}$ \\ Erika Renata Nascimento Cavalcanti de Oliveira ${ }^{4}$
}

\footnotetext{
${ }^{1}$ Psicólogo residente em cuidados paliativos. Escola Superior de Ciências da Saúde - ESCS/FEPECS

${ }^{2}$ Enfermeira, MSc, coordenadora do Programa de Residência Multiprofissional em Cuidados Paliativos

${ }^{3}$ Psicóloga do Hospital de Apoio de Brasília - SES/DF

${ }^{4}$ Médica do Hospital de Apoio de Brasília - SES/DF
}

\section{RESUMO}

Os cuidados paliativos diante da pandemia pelo COVID-19 terão importância ímpar e grandes desafios na reorganização dos seus dispositivos para o cuidado. Este artigo apresenta uma proposta de estruturação de atendimento de cuidados paliativos na geriatria para o manejo de idosos frágeis com diagnóstico confirmado ou suspeito de coronavírus e sem indicação de medidas sustentadoras da vida. Logo, discutir-se-á a tomada de decisão, manejo de sintomas e a atuação da equipe de cuidados paliativos junto com o paciente e família, analisando os benefícios dessa modalidade de cuidado.

Palavras-chave: cuidados paliativos, COVID-19, idosos, manejo de sintomas, atuação da equipe.

\section{Palleative care for fragile elderly during Coronavirus pandemic}

\begin{abstract}
Palliative care during COVID-19 pandemic will have unparalled importance and bring challenges to rearrange its methods of care. This article proposes a model of palliative care treatment in geriatrics to the management of fragile elderly that have suspected or confirmed diagnosis of coronavirus without any indication for life sustaining measures. Therefore, it discusses decision making, symptom management and palliative care team work with the patient and his family, analysing the benefits of the model.
\end{abstract}

Key words: palliative care, COVID-19, elderly, symptom management, team work. 


\section{INTRODUÇÃO}

O Sars-COV-2, da família dos coronavírus, foi detectado em 31 de dezembro na China. No Brasil, o primeiro caso confirmado foi em 26 de fevereiro no estado de São Paulo, chegando em Brasília em 07 de março ${ }^{1}$. Diante da transmissibilidade de 1,6 a 4,1 e letalidade de 2,2\% no geral e 13,2\% entre a população mais frágil, o vírus SARS-COV-2 tornou-se um agravo para a saúde pública ${ }^{2}$.

Atualmente, até o dia 27 de fevereiro de 2021 são 113.784 .735 casos confirmados e 2.525.401 óbitos no mundo. No Brasil, até a mesma data, foram confirmados 10.517.232 casos de COVID-19 e 254.221 óbitos. O Distrito Federal, décima região com maior incidência por 100 mil habitantes, já confirmou 338.930 casos e 5.717 óbitos ${ }^{3,4}$.

Mundialmente, a pandemia se configura com maior virulência entre idosos com mais de 70 anos e indivíduos com maior comorbidade e fragilidade ${ }^{5}$. Há também evidências de que $15 \%$ dos infectados apresentarão quadro grave da doença com necessidade de terapias de sustentação de vida como a intubação, ventilação mecânica, hemodiálise e vasopressores ${ }^{6}$.

Nesse sentido, estar diante de uma população idosa, fragilizada pelas comorbidades múltiplas e com uma infecção aguda e potencialmente letal nos obriga a pensar desde o início do processo de adoecimento, no tratamento e na tomada de decisão diante do doente e família ${ }^{7}$. O atendimento, portanto, deve ser sistemático com manejo dos sintomas, centrado no paciente e com avaliação equilibrada, compartilhada e cuidadosamente discutida pela equipe e família.

No adoecimento pelo COVID-19 em indivíduos vulneráveis, o sofrimento é múltiplo. Há desde o manejo difícil dos sintomas físicos (fadiga e dispneia), o medo e ansiedade decorrente de uma doença recente e fatal até a falta de informação e separação da família. Todas essas condições são exacerbadas pela capacidade limitada do sistema de saúde no 
atendimento, o que provavelmente acarretará uma angústia espiritual diante do sentimento de abandono e a desesperança ${ }^{7}$.

Assim, a partir de demandas complexas, o cuidado paliativo cresce em significado diante dessa população. O IAHPC (International Association for Hospice \& Palliative Care) definiu, em 2018, os cuidados paliativos como cuidado multidimensional ativo dos indivíduos com graves sofrimentos relacionados à saúde, independentemente da faixa etária ${ }^{8}$. Essa modalidade de cuidado, a partir dos profissionais com habilidades clínicas para o gerenciamento dos sintomas e comunicação, acolherá com o objetivo de proporcionar maior bem-estar, conforto e dignidade, especialmente no fim de vida

Deve-se atentar, no entanto, que as equipes de cuidados paliativos e os serviços são escassos ${ }^{9}$. Corroborando com esse dado, em avaliação do índice de morte, em 2015 o Brasil estava na 42 qualificação, quando se avaliava a acessibilidade, qualidade no atendimento, recursos humanos, acesso a medicações opioides e contexto de cuidados paliativos em saúde ${ }^{10}$.

Nesse sentido, surge a importância de dinamizar o serviço criando diretrizes de estruturação, algoritmos práticos para apoiar a equipe interdisciplinar da linha de frente que respondem ao COVID-197. Além disso, espera-se que as unidades de cuidados paliativos possam cooperar com o atendimento de pacientes com doenças graves e ameaçadoras de vida com baixa reserva funcional e deterioração clínica.

Nessa perspectiva, a câmara técnica de Cuidados Paliativos do Distrito Federal, o qual encontra-se em posição central, assume sua responsabilidade diante da complexidade de saúde advinda da pandemia e cria diretrizes de cuidado. Essa equipe interdisciplinar, portanto, cria nota técnica que visa abordar os principais tópicos em situação de pandemia por COVID 19, que são: a comunicação, tomada de decisão, controle de sintomas e autocuidado dos profissionais de Saúde ${ }^{11}$ e que serão discutidas ao longo do texto. 
Logo, a população e as necessidades atendidas nos cuidados paliativos vão ao encontro das demandas holísticas e interdisciplinares do doente com COVID-19 ${ }^{12}$. Assim, o objetivo do estudo é demonstrar uma proposta de atendimento baseado nos cuidados paliativos geriátricos em idosos com diagnóstico suspeito ou confirmado de coronavírus e sem possibilidade/indicação de medidas que prolonguem a vida de forma artificial, discutindo a atuação da equipe de cuidados paliativos junto com o paciente e família e analisando os benefícios dessa modalidade de cuidado.

\section{A TOMADA DE DECISÃO NOS CUIDADOS PALIATIVOS}

Atender pacientes com a proposta de confortar e evitar a obstinação terapêutica deverá ter como princípios norteadores o alívio da dor e demais sintomas angustiantes, integrar aspectos sociais e psicoespirituais no cuidado, afirmar a vida e reconhecer a morte como um processo natural e oferecer um sistema de suporte junto a família que permita o doente viver ativamente, tanto quanto possível, até a morte ${ }^{13}$.

Nesse sentido, todas as pessoas (independentemente da suspeita ou confirmação do COVID-19) devem ter atendimento nesse modelo de cuidado. Há, no entanto, critérios para a decisão de não prolongar a vida, evitando métodos fúteis e alocando recursos físicos e humanos de uma forma justa. Essa decisão, todavia, deve ser metódica e baseada em princípios, considerando a gravidade da doença do paciente, sua história de vida, escolhas, funcionalidade e sua família. É necessário também atentar para aqueles que não foram afetados pela pandemia, na medida em que o racionamento de recursos não pode ter como consequência o abandono destes pacientes.

Para tanto, é necessário a definição de doença grave. Essa é definida como risco de vida acompanhada por sintomas de difícil controle ou que alteram drasticamente a qualidade de vida da pessoa ${ }^{13}$. No idoso, muitas vezes acometido por síndromes, é fundamental a 
estratificação clínica não se restringindo a idade, mas sim a avaliação da vitalidade, ou seja, a reserva homeostática do idoso com a capacidade de reagir a uma infecção como a do coronavírus.

Nessa avaliação deve-se considerar a funcionalidade do idoso antes da infecção do SARS-CoV-2. Assim, o presente artigo sugere a avaliação a partir do IVCF-20. Essa escala sugerida para avaliação do idoso, consta de 20 questões e escore máximo de 40 pontos. Examina-se a capacidade funcional, a partir da independência nas atividades de vida diária básicas (transferir, continência, alimentar-se, banhar-se, vestir-se) e instrumentais (preparar a refeição, usar o telefone, fazer compras, medicar-se...) com classificação do idoso em 10 estratos $^{14}$.

Para fins práticos, idosos frágeis que têm estrato 10,9 ou 8 ou que pontuaram na escala mais de 15 pontos devem ter atenção e intervenção de equipe especializada em cuidados paliativos. Esses teriam como proposta de atendimento um plano avançado de cuidados, contando com equipe interdisciplinar para manejo de sintomas.

No tocante à essa decisão, alguns estudos sugerem outros indicadores para a identificação rápida que o doente se aproxima da fase final de vida e necessidade emergente dos cuidados paliativos exclusivos. O primeiro delas trata-se da avaliação profissional onde é feita a pergunta: "você ficaria surpreso se o paciente falecesse nos próximos dias ou semanas?" Esse questionamento, apesar da baixa sensibilidade e especificidade em pacientes sem câncer, associado com outros indicadores poderá contribuir com a decisão clínica de atendimento por uma equipe interdisciplinar terciária especializada em casos complexos ${ }^{15,16}$.

O fato é que os principais indicadores gerais de declínio que são propostos por guidelines são o declínio físico e da funcionalidade ${ }^{17}$. Há ainda escalas validadas, como o SPICT (Supportive and Palliative Care Indicators Tool), que auxiliam na tomada de decisão quanto a proporcionalidade do atendimento. Nesse instrumento, permanece o destaque para a 
funcionalidade e estado físico e acrescenta indicadores clínicos nos múltiplos adoecimentos como forma de nortear o propósito do cuidado ${ }^{18}$.

Assim, a partir da suspeita ou confirmação do diagnóstico a tomada de decisão quanto aos cuidados paliativos é necessária e a comunicação e manejo dos sintomas se atentando para o binômio doente e família é indispensável ${ }^{11}$. Nesse sentido far-se-á a discussão quanto aos principais sintomas relatados por doentes contaminados por Sars-COV-2, como também as melhores técnicas para o seu manejo. Posteriormente, será exposto a atuação da equipe junto a família discutindo aspectos importantes sobre comunicação, empatia e sabedoria no ato de acolher e comunicar.

\section{GERENCIAMENTO DE SINTOMAS}

Diante do adoecimento agudo e evolução rápida para insuficiência respiratória no coronavírus, torna-se indispensável o reconhecimento e manejo de sintomas desconfortantes para qualidade de vida. A partir do manejo dos sintomas, os pacientes poderão ter melhor experiência durante a doença como também ter maior dignidade e conforto na morte.

Quanto ao manejo dos sintomas no COVID-19, deve-se atentar que o fim de vida pode ser rápido, durando poucas horas (principalmente na população idosa) ${ }^{19}$. Os sintomas de dispneia, tosse, delirium e agitação, comuns nessa etapa se desenvolvem rapidamente e podem ser muito angustiantes ${ }^{20}$. Outra revisão aponta que os sintomas mais comuns relatados são febre, tosse, mialgia ou fadiga, dispneia complicada, enquanto sintomas relatados menos comuns incluem dor de cabeça, diarreia, hemoptise, coriza e tosse ${ }^{21}$. Nesse sentido, o rápido acesso ao tratamento farmacológico é vital e geralmente pode envolver doses maiores à prática padrão dos cuidados paliativos ${ }^{22}$.

Considerando o dever ético de aliviar esses sintomas angustiantes, algumas classes de fármacos podem ser usadas no manejo (mesmo daqueles sem nenhuma chance de cura). Entre 
elas estão analgésicos e anti-térmicos para o tratamento da hipertermia e adjuvantes da dor e os opióides para manejo da dispneia e dor moderada a intensa. Além disso, há os antipsicóticos que devem ser necessários para as consequências cognitivas do delirium e agitação e os anticolinérgicos para tratamento de secreção de vias aéreas ${ }^{11,23-25}$.

Todo o manejo farmacológico torna-se efetivo, no entanto, com a participação e protagonismo da equipe multidisciplinar. Ações relacionadas ao posicionamento, apoio emocional e monitoramento de complicações como lesão por pressão, broncoaspiração e edema ganham importância e são imprescindíveis ${ }^{26}$. O fato é que a oxigenação prejudicada causada por uma lesão pulmonar decorrente da inflamação pelo COVID-19 traz um grande desafio à função cardiopulmonar, especialmente para os idosos ${ }^{19}$.

Diante dessa premissa, organizaram-se sete tópicos importantes para o manejo não farmacológico dos sintomas e maior conforto do doente ${ }^{11}$.

1. Avaliar frequentemente os sintomas a partir de escala

2. Reposicionar frequentemente o paciente

3. Apoiar emocionalmente o paciente e família

4. Revisar e interromper dispositivos invasivos que provoquem desconforto;

5. Revisar e interromper líquidos endovenosos e orais;

6. Monitorar complicações como lesão por pressão, broncoaspiração e edema

7. Considerar o início da terapia subcutânea

\section{ATUAÇÃO DA EQUIPE}

Quanto à atuação da equipe, destaca-se a comunicação empática, que é fundamental no resultado ao lidar com o paciente e sua família. Apesar de não haver consenso sobre seu significado, a empatia pode ser descrita como a capacidade de perceber os significados e sentimentos do outro e comunicá-los a ele $^{27}$, imaginando a situação pelo ponto de vista da outra pessoa. Algumas características dessa comunicação são: identificar a perspectiva do 
outro; não julgar; reconhecer as emoções do outro; comunicar o que foi percebido. A comunicação adequada pode reduzir a angústia e ansiedade da família, melhorando a adaptação ao plano terapêutico. Outras fontes adicionam o conceito de compaixão $^{28}$, na medida em que ela complementaria a empatia com ser motivado pelo amor e o papel altruísta do agente que atua de forma bondosa.

No sentido de subsidiar a comunicação de assuntos delicados, há o protocolo de comunicação de más notícias SPIKES ${ }^{29}$, que tem seis etapas. A primeira consiste em planejar a entrevista, seguido por avaliar a percepção do paciente, obter seu convite para a conversa, dar conhecimento a ele, abordar suas emoções com respostas afetivas e então discutir as estratégias do tratamento e resumí-las. Um protocolo adaptado ao contexto brasileiro utilizase da sigla PACIENTE ${ }^{30}$, sendo bastante similar ao SPIKES e adicionando a necessidade de não abandonar o paciente.

O fato é que o suporte psicossocial é tão importante quanto o controle da infecção durante a pandemia ${ }^{31}$. Tendo como objetivo fornecer esse tipo de apoio a pacientes e familiares seria uma boa opção a produção de panfletos que fizessem um resumo de informações importantes (que se utilizem de uma comunicação empática e possam ser facilmente entendidas e colocadas em prática).

Nessa perspectiva, a Sociedade Coreana de Estudos sobre Estresse Traumático criou um manual de orientações das mais variadas às mais específicas ${ }^{32}$. Trazendo para a realidade da pandemia, algumas informações importantes que poderiam ser abordadas. Entre elas estão as possíveis dificuldades emocionais envolvidas no processo de adoecimento ou acompanhamento de uma pessoa infectada; quais atividades se engajar para manejo de estresse (atividades físicas, lúdicas, relaxantes) e quais se distanciar (ler muitas notícias, desregular o ciclo de sono e outras); formas de despedir-se da pessoa em processo de morte, como por chamada de vídeo ou presencialmente usando EPIs; focar em lembrar dos 
momentos bons que se passou com a pessoa; até mesmo sobre como a equipe de saúde deve proceder diante da morte e do luto.

Adicionalmente, é necessário que se fale também de luto. $\mathrm{O}$ enfrentamento ao luto envolve respostas emocionais, cognitivas, físicas e comportamentais comuns apósperdas ${ }^{33}$. Atualmente o ser humano está exposto a vários tipos de perdas: financeiras, sociais, autonômicas e outras. O luto antecipatório é um fenômeno normal quando há a expectativa da morte, sendo caracterizado por conjunto de mecanismos de enfrentamento utilizados pela família e paciente frente à possibilidade de perda antecipada ${ }^{33}$, ou seja: é uma experiência similar ao luto, mas que é vivida de forma gradual e enquanto a pessoa ainda está viva. O aumento vertiginoso de mortes pelo vírus faz com que profissionais de saúde, pacientes e familiares vivenciem incertezas e tentem dar sentido ao que acontece diariamente e ao que é esperado do futuro.

Portanto, ratifica-se a necessidade de acompanhamento pela equipe de saúde do luto antecipatório, minimizando então os riscos de luto complicado. A condição de urgência em saúde, no entanto, torna difícil a organização de cenários favoráveis à vivência monitorada desse luto. Dessa forma, é importante que a equipe se prepare e disponibilize espaços para acompanhamento do processo de luto pós-óbito do paciente.

Considerando a situação, também é fundamental que os profissionais de saúde compreendam quais os contextos e sintomas favoráveis para o desenvolvimento de luto complicado. Os sintomas podem $\operatorname{ser}^{34}$ : pensamentos intrusivos recorrentes sobre a pessoa perdida; preocupação com sofrimento e pensamentos ruminantes; ressentimento exagerado; afastamento de relaçoes sociais anteriores; dificuldade em aceitar a morte; falta de sentido da vida. A incapacidade de se despedir, ter baixos níveis de suporte social e falta de preparação para a morte também estão relacionados com o luto complicado, assim como o tipo e a quantidade de mortes. Desse modo, deve-se atentar para o atendimento psicoespiritual do 
familiar, levando em conta ritos de passagem e tradições culturais e religiosas ${ }^{35}$, já que no contexto de tratamento do coronavírus todos esses sintomas têm alta probabilidade de ocorrência.

Por último, é importante discutir a atuação dos profissionais de saúde envolvidos nesse cuidado, onde muitos se utilizam de mecanismos de defesa como a evitação ou compartimentalização para continuar tratando seus pacientes, o que pode levar a lutos mal resolvidos. É comum que essas pessoas sofram de estresse traumático secundário, que é uma resposta de estresse que ocorre ao conhecer ou ajudar uma pessoa que vive um trauma, e é caracterizado pelos seguintes sintomas: medo e preocupação excessiva; sentir-se "de guarda" o tempo todo; pensamentos recorrentes; sinais físicos de estresse ${ }^{34}$.

Esses profissionais precisam se autoconhecer, compreender e falar sobre seus próprios sentimentos e emoções e, além disso, dar pausas no trabalho para se desconectar dos eventos, sentir-se preparado e informado para atuar de forma eficiente, estar ciente dos recursos locais e serviços de referência e ter supervisão adequada e suporte de seus colegas ${ }^{34}$. $\mathrm{O}$ autocuidado também é evidenciado como uma medida importante para o resultado do tratamento. Algumas práticas que podem aliviar o luto, luto complicado e a atuação dos profissionais estão relacionadas a comunicação de qualidade e conversar sobre as diretivas antecipadas de vontade $^{27}$. Considerando as restrições a visitas, é necessário discutir a possibilidade das mesmas em quais condições e, caso seja impossível, sobre outras alternativas (como as chamadas ou videochamadas ${ }^{36}$ ).

\section{CONCLUSÕES}

A proposta inicial do artigo se manifesta como relevante na medida em que equipes profissionais brasileiras podem estar abaladas mediante o contexto da pandemia. Nesse sentido, modelos de intervenção (tanto gerais como específicos) devem ser desenvolvidos nos 
mais diversos âmbitos para que se seja capaz de atender às demandas recentes. Um exemplo disso pode ser visto no manual produzido por equipe sul-coreana ${ }^{31}$, onde são encontradas orientações também para idosos e familiares.

A especificidade da população (idosos fragéis) aliada à descrição sintomatológica nacional e internacional ${ }^{19-22}$ torna possível a formulação de um modelo de cuidados característico, mas que pode ser adaptado ou complementado levando em consideração outras variáveis não discutidas neste texto. É primordial esclarecer que essa proposta, apesar de teoricamente coesa, deve ser testada na prática, afim de determinar sua validade e adequação em cada cenário hospitalar e de saúde propriamente dito.

A abordagem de cuidados paliativos se mostra basilar, tanto para o tratamento de COVID-19 como para a abordagem em geral na saúde, porque tem como foco o bem-estar, conforto e dignidade dos pacientes e não somente a cura ${ }^{27}$. Esse tipo de intervenção deve ocorrer conjuntamente aos esforços para tratar a infecção e salvar vidas, podendo até mesmo melhorar a sobrevivência. A resposta à disseminação do covid-19 fez com que várias sociedades adotassem políticas de isolamento e distanciamento social (muitas vezes de forma obrigatória) e, enquanto as mortes ocorrem, os efeitos físicos, mentais e sociais desses isolamentos podem influenciar a saúde mental dos envolvidos e o potencial de desenvolvimento de lutos complicados ${ }^{33}$. O enfoque, portanto, não deve ser somente na cura e sim em prover cuidado ao paciente e à família, independentemente da possibilidade de cura, de forma a aliviar todos os malefícios provenientes da situação em que se encontra o mundo atualmente. 


\section{REFERÊNCIAS}

1. Secretaria de Vigilância em Saúde M, Saúde D. Infecção humana pelo novo coronavírus (2019-nCoV). Boletim Epidemiológico 2020; (08) [Internet]. 2020. Available from: https://www.saude.gov.br/images/pdf/2020/April/06/2020-04-06-BE7-BoletimEspecial-do-COE-Atualizacao-da-Avaliacao-de-Risco.pdf

2. Lana RM, Coelho FC, Gomes MF da C, Cruz OG, Bastos LS, Villela DAM, et al. Emergência do novo coronavírus (SARS-CoV-2) e o papel de uma vigilância nacional em saúde oportuna e efetiva. Cad Saude Publica. 2020;36(3):e00019620.

3. Secretaria de Saúde DF. Boletim Epidemiológico n 390: Emergência de Saúde Pública COVID-19 no âmbito do Distrito Federal. 2021.

4. Ministério da S. Boletim epidemiológico especial 52: Doença pelo Coronavírus COVID19. 2021 p. 1:85.

5. Kolifarhood G, Aghaali M, Saadati HM, Taherpour N. Epidemiological and Clinical Aspects of COVID-19; a Narrative Review. Arch Acad Emerg Med. 2020;8(1):1-9.

6. Murthy S, Gomersall CD, Fowler RA. Care for Critically Ill Patients with COVID-19. JAMA - J Am Med Assoc. 2020;1-2.

7. Brynn A. Bowman, Andrew E. Esch, Anthony L. Back NM. Crisis Symptom Management and Patient Communication Protocols Are Important Tools for All Clinicians Responding to COVID-19. J Pain Symptom Manage. 2020;(January).

8. Definition IA for H\& PCPC. No Title. 2018.

9. Academia Nacional de Cuidados Paliativos. Análise Situacional e Recomendações Para Estruturação De Programas De Cuidados Paliativos No Brasil [Internet]. Academia Nacional de Cuidados Paliativos. 2018. 28 p. Available from: https://paliativo.org.br/wpcontent/uploads/2018/12/ANALISE-SITUACIONAL_ANCP-18122018.pdf

10. The Economist Intelligence Unit. The 2015 Quality of Death Index Ranking palliative 
care across the world. The Economist [Internet]. 2015;71. Available from: http://www.apcp.com.pt/uploads/2015-EIU-Quality-of-Death-Index-Oct-6-FINAL.pdf

11. Paliativos CT de C. Nota técnica de cuidados paliativos: Abordagens imprescindíveis em Cuidados Paliativos em situação de pandemia por COVID 19: Comunicação, Tomada de Decisão, Controle de sintomas e Autocuidado dos profissionais de Saúde. 2020 p. 1:17.

12. Stow D, Spiers G, Matthews FE, Hanratty B. What is the evidence that people with frailty have needs for palliative care at the end of life? A systematic review and narrative synthesis. Palliat Med. 2019;33(4):399-414.

13. Finn L, Malhotra S. The Development of Pathways in Palliative Medicine: Definition, Models, Cost and Quality Impact. Healthcare. 2019;7(1):22.

14. Moraes EN, Carmo JA, Moraes FL, Azevedo RS, Machado CJ, Montilla DER. Clinical-Functional Vulnerability Index-20 (IVCF-20): Rapid recognition of frail older adults. Rev Saude Publica. 2016;50:1-10.

15. Downar J, Goldman R, Pinto R, Englesakis M, Adhikari NKJ. The "surprise question" for predicting death in seriously ill patients: A systematic review and meta-analysis. Cmaj. 2017;189(13):E484-93.

16. White N, Kupeli N, Vickerstaff V, Stone P. How accurate is the "Surprise Question" at identifying patients at the end of life? A systematic review and meta-analysis. BMC Med. 2017;15(1):1-14.

17. Thomas J, Wilson A. The Gold Standards Framework Proactive Identification Guidance - The National GSF Centre's guidance for clinicians to support earlier identification of patients nearing the end of life leading to improved proactive person-centred care [Internet]. Royal College of General Practioners. 2016. Available from: http://www.goldstandardsframework.org.ukformoredetailsseegsfpig

18. De Bock R, Van Den Noortgate N, Piers R. Validation of the Supportive and Palliative 
Care Indicators Tool in a Geriatric Population. J Palliat Med. 2018;21(2):220-4.

19. Lang W, Wenbo H, Xiaomei Y, Dalong H, Mingwei B, Huafen L, et al. Coronavirus disease 2019 in elderly patients: Characteristics and prognostic factors based on 4-week follow-up. J Infect. 2020;5G(January):11:20.

20. Liu K, Chen Y, Lin R, Han K. Clinical features of COVID-19 in elderly patients: A comparison with young and middle-aged patients. J Infect [Internet]. 2020;80(6):e14-8. Available from: https://doi.org/10.1016/j.jinf.2020.03.005

21. Wynants L, Van Calster B, Bonten MMJ, Collins GS, Debray TPA, De Vos M, et al. Prediction models for diagnosis and prognosis of covid-19 infection: Systematic review and critical appraisal. BMJ. 2020;369.

22. Pallipedia I. Guidance on the management of symptomatic patients dying from COVID-19.

https://elearning.rcgp.org.uk/pluginfile.php/149344/mod_resource/content/1/Covid\%20eolc\% 20community\%20symptom\%20management $\% 2027 \% 2003 \% 202020 \% 20$ final.pdf.pdf Accessed on March 1, 2020. 2020. p. 1:3.

23. Eriksen S, Grov EK, Lichtwarck B, ... Palliative treatment and care for dying nursing home patients with COVID-19. Tidsskr Den Nor legeforening [Internet]. 2020; Available from: https://tidsskriftet.no/en/2020/05/klinisk-oversikt/palliative-treatment-and-care-dyingnursing-home-patients-covid-19

24. Roland K, Markus M. COVID-19 pandemic: Palliative care for elderly and frail patients at home and in residential and nursing homes. Swiss Med Wkly. 2020;150(1314):0-1.

25. Care TIA for $\mathrm{H}$ and P. Recommendations for Symptom Control of Patients with COVID-19. The International Association for Hospice and Palliative Care. 2020.

26. Hendin A, La Rivière CG, Williscroft DM, O’Connor E, Hughes J, Fischer LM. End- 
of-life care in the Emergency Department for the patient imminently dying of a highly transmissible acute respiratory infection (such as COVID-19). CJEM. 2020;1(4):1-5.

27. Carvalho RT, Souza MRB, Franck EM, Polastrini RTV, Crispim D, Jales SM, et al. Manual da residência de cuidados paliativos. 2018.

28. Sinclair S, Beamer K, Hack TF, McClement S, Raffin Bouchal S, Chochinov HM, et al. Sympathy, empathy, and compassion: A grounded theory study of palliative care patients' understandings, experiences, and preferences. Palliat Med. 2017;31(5):437-47.

29. Baile WF, Buckman R, Lenzi R, Glober G, Beale EA, Kudelka AP. SPIKES - Um Protocolo em Seis Etapas para Transmitir Más Notícias : Aplicação ao Paciente com Câncer. Oncologist. 1998;1-14.

30. Pereira CR, CalôNego MAM, Lemonica L, De Barros GAM. The P-A-C-I-E-N-T-E Protocol: An instrument for breaking bad news adapted to the Brazilian medical reality. Rev Assoc Med Bras. 2017;63(1):43-9.

31. Hyun J, You S, Sohn S, Kim SJ, Bae J, Baik M, et al. Psychosocial Support during the COVID-19 Outbreak in Korea: Activities of Multidisciplinary Mental Health Professionals. J Korean Med Sci [Internet]. 2020 Jun 8;35(22):e211. Available from: http://www.ncbi.nlm.nih.gov/pubmed/32508070

32. Korean Society for Traumatic Stress Studies. (2020). Guidelines on psychosocial care for infectious disease management. Gyeongsan: KSTSS. Available from: http://kstss.kr/?page_id=1534

33. Santos RCS, Yamamoto YM, Custódio LMG. Aspectos Teóricos Sobre O Processo de Luto e a Vivência do Luto Antecipatório. 2017;1-18. Available from: http://www.psicologia.pt/artigos/textos/A1161.pdf

34. Wallace CL, Wladkowski SP, Gibson A, White P. Grief During the COVID-19 Pandemic: Considerations for Palliative Care Providers. J Pain Symptom Manage [Internet]. 
2020; Available from: https://pubmed.ncbi.nlm.nih.gov/32298748

35. Alleviating suffering and upholding dignity in the midst of CoViD-19 response: A place for palliative care Palliative care is an integral component of pandemic response plans and activities. $\quad$ R2HC $\quad$ Internet]. 2020. Available from: https://www.phpc.cam.ac.uk/pcu/files/2020/04/PallcareCovid_Recommendations_FINAL.pdf 36. Etkind SN, Bone AE, Lovell N, Cripps RL, Harding R, Higginson IJ, et al. The Role and Response of Palliative Care and Hospice Services in Epidemics and Pandemics: A Rapid Review to Inform Practice During the COVID-19 Pandemic. J Pain Symptom Manage [Internet]. 2020; Available from: https://doi.org/10.1016/j.jpainsymman.2020.03.029 